\title{
Validez y confiabilidad de un instrumento de satisfacción del usuario con síndrome febril agudo
}

The validity and reliability of an instrument for measuring the satisfaction of users suffering from acute febrile syndrome

\author{
Diana C. Tiga-Loza, Luis Á. Villar-Centeno, Diana R. Güiza-Sanabria y \\ Ruth A. Martínez-Vega \\ Universidad Industrial de Santander. Bucaramanga, Colombia. caritotiga@hotmail.com, \\ luisangelvillarc@yahoo.com,dirock_44@hotmail.com,rutharam@yahoo.com \\ Recibido 24 Febrero 2010/Enviado para Modificación 12 Diciembre 2010/Aceptado 27 Diciembre 2010
}

\section{RESUMEN}

Objetivo Determinar la validez y la confiabilidad de un instrumento de satisfacción del paciente con Síndrome Febril Agudo en urgencias y consulta oportuna de la Red Pública de Bucaramanga.

Métodos Estudio de evaluación de tecnologías diagnósticas en personas con Síndrome Febril Agudo entre el 2008 y 2009. Se diseñó un cuestionario telefónico a partir de 3 instrumentos en español. Se evaluó validez de contenido mediante sometimiento a expertos y a profesionales de salud y validez facial en una prueba piloto con 30 usuarios. La reproducibilidad fue realizada por dos enfermeras independientemente en 19 encuestas.

Resultados Se realizaron 146 encuestas, de estas $75.3 \%$ fueron contestadas por mujeres, $33.6 \%$ por los mismos pacientes y $52.7 \%$ por las madres de estos cuando eran menores de 15 años. Se realizó análisis factorial obteniendo 3 factores: satisfacción con la atención médica, de enfermería y la organización del centro; al modelo final entraron 16 de 26 ítems. El alfa de Cronbach para cada factor fue 0,89 (IC $95 \%$ : 0,86;0,93), 0,78 (IC95\%: 0.67;0.84) y 0,88 (IC95\%: $0.85 ; 0.91$ ) respectivamente; el coeficiente de kappa fue 0.90 (IC95\%: 0.77;0.97) y el coeficiente de correlación intraclase fue 0.955 (IC95\%: 0.862;0.986) El promedio de las diferencias en la sumatoria de los ítems fue -1.3 (DS: 8,5) y los límites de acuerdo de Bland y Altman fueron -18.9 y 15.3.

Discusión Este estudio demuestra validez y confiabilidad del instrumento de satisfacción de usuarios de los servicios de urgencias y consulta oportuna y podría aplicarse en la red pública del sistema de salud Colombiano.

Palabras Clave: Satisfacción del paciente, urgencias médicas, fiebre, Reproducibilidad de Resultados, validez de las pruebas (fuente: DeCS, BIREME).

\section{ABSTRACT}

Objective Determining the validity and reliability of a user satisfaction instrument applied to patients attending the emergency department and clinic who were suf- 
fering from acute febrile syndrome in Bucaramanga's Public Health Network. Methods A diagnostic technology assessment study was conducted between 2008 and 2009 on patients suffering from acute febrile syndrome. A telephone questionnaire was designed using 3 instruments. Content validity was assessed by experts and health professionals and face validity was assessed in a pilot study involving 30 users. Reproducibility was tested in questionnaires independently applied by two nurses to 19 patients.

Results Data from 146 surveys was collected; $75.3 \%$ were answered by women, $33.6 \%$ were patients and $52.7 \%$ were their mothers when patients were under 15 . Three factors were identified from factor analysis: satisfaction with medical care, nursing and medical centre organisation. The final model included 16 of the 26 items. Cronbach's Alpha for each factor was $0.89(0.86-0.9395 \% \mathrm{Cl}), 0.78(0.67-$ $0.8495 \% \mathrm{Cl})$ and $0.88(0.85-0.9195 \% \mathrm{Cl})$, respectively. The Kappa coefficient was $0.90(0.77-0.9795 \% \mathrm{Cl})$ and intra-class correlation coefficient was $0.96(0.86-0.99$ $95 \% \mathrm{Cl}$ ). The average difference of the sum of all items was -1.3 (SD: 8.5$)$ and Bland and Altman limits of agreement were -18.9 and 15.3, respectively.

Discussion This study established the validity and reliability of a new instrument concerning user satisfaction used with patients suffering from acute febrile syndrome attending emergency health care which that could be applied in the Colombian Public Health System.

Key Words: Patient satisfaction, emergency, fever, reproducibility of results, reliability (source: MeSH, NLM).

a satisfacción del usuario de los servicios de salud es considerada como una medida importante de resultado para evaluar la calidad de la atención en salud y está definida como el grado de concordancia (conformidad o discrepancia) entre las expectativas y la percepción final del servicio recibido $(1,2)$. Así mismo, la satisfacción está relacionada con el uso de los servicios y los cambios en el estado de salud de las personas atendidas $(3,4)$; a su vez, ésta se ha convertido en un importante indicador en la evaluación y mejoramiento de la calidad en la atención de los servicios de salud $(5,6)$, pues permite evaluar la consulta prestada, los patrones de comunicación entre el usuario y el personal y orienta la elección de formas de organización y prestación de los servicios de salud $(7,8)$. Una forma de medir la satisfacción de la persona atendida es utilizando encuestas de opinión, las cuales permiten ver las percepciones sobre la atención prestada y así detectar aspectos susceptibles de mejora, insuficiencias o disconformidades (9).

En concordancia con lo anterior, en el 2006 el Ministerio de Protección Social en Colombia, estableció de manera obligatoria la medición de la satisfacción global como indicador de calidad dentro del Sistema Obligatorio de la Garantía de la Calidad en Salud (SOGCS) y determinó como mecanismo de medición las encuestas de satisfacción de los usuarios (10). 
En las últimas décadas se han elaborado numerosos cuestionarios para medir la satisfacción de los pacientes en diferentes aéreas de la salud $(11,12)$ sin embargo, se encuentran en la literatura pocos instrumentos válidos y reproducibles en el idioma español (13-15); En Colombia, se han aplicado instrumentos para evaluar la satisfacción de los pacientes en odontología (16), fisioterapia, nutrición, fonoaudiología y terapia respiratoria (17) y para evaluar la calidad de algunas técnicas anestésicas y su recuperación (1819), sin embargo, sólo en uno de ellos fueron evaluadas las características psicométricas del instrumento (19).

El servicio de urgencias es considerado como uno de los que maneja mayores niveles de tensión, con ritmos de trabajo exigentes y acompañados de alta afluencia de pacientes; factores que pueden incidir en el nivel de satisfacción percibido por el usuario (20). Además el servicio de urgencias es el segundo más frecuentado después de consulta externa. En el ámbito nacional, $17 \%$ del volumen de atención de las Instituciones Prestadoras de Salud corresponde a la atención de urgencias (21) y en Santander 5,9\% de los Registros Individuales de Prestación de Servicios de Salud (RIPS) del régimen contributivo pertenecen a urgencias (22). Adicionalmente, el Síndrome Febril Agudo (SFA) es uno de los diagnósticos más prevalentes en los servicios de consulta oportuna en Bucaramanga (22). Este estudio pretende determinar la validez de contenido y la confiabilidad de un instrumento para medir satisfacción del usuario con SFA que acude a los servicios de urgencias y consulta oportuna de Bucaramanga, de manera que constituya en una herramienta útil y estandarizada para determinar la percepción de calidad y a su vez permita la comparabilidad de los resultados entre las diferentes instituciones o servicios.

\section{MATERIALES Y MÉTODOS}

Diseño

Estudio de evaluación de tecnologías diagnósticas (23).

Población y Muestra

Para la validación de este instrumento fueron encuestadas aquellas personas con SFA mayores de 15 años o los representantes legales y acudientes de los menores de 15 años que asistieron al servicio de urgencias del Hospital Local del Norte o a consulta prioritaria de los centros de salud Rosario, Toledo Plata, Girardot y Mutis durante los años 2008 y 2009. Se excluyeron las personas 
que no tenían un número telefónico o el teléfono suministrado no correspondía, las que tenían discapacidad auditiva o mental y consentimiento verbal negativo.

\section{Construcción del instrumento}

Se realizó una búsqueda de la literatura para identificar estudios que hubiesen desarrollado cuestionarios de satisfacción, en especial en idioma español, encontrándose 3 artículos que se complementaban entre sí, abarcando el máximo de dimensiones de satisfacción: Cuestionario 1. Encuesta telefónica URGENSA 1.2 con 16 ítems en su versión reducida (13); Cuestionario 2. Escala SERCAL modificación de SERVQUAL (servicio confortable, accesible personalizado, de garantía), compuesto por 20 ítems, 18 de los cuales eran medidos con una escala tipo likert que iba de 0 a 10 (total desacuerdo o insatisfacción a total acuerdo o satisfacción) y un ítem dicotómico (si, no) y otro ordinal (peor mejor igual) (14); Cuestionario 3. Instrumento utilizado en la Encuesta a Usuarios de los servicios de atención primaria del Servicio Andaluz de Salud (SAS) con escala tipo Likert de 5 puntos y 1 ítem dicotómico (15). Con base en estos instrumentos, se construyó uno compuesto por 26 ítems utilizando una escala de 0 a 10 en el cual 0 significa "Nada satisfecho" y 10 "Totalmente satisfecho"; 7 de los ítems evaluaban la satisfacción con la atención médica, 5 la atención de enfermería, 3 la atención del personal administrativo, 5 las instalaciones, 5 la organización del centro de atención en salud y 1 de satisfacción general. En el Apéndice 1 se encuentra el instrumento utilizado.

Posteriormente, el instrumento fue sometido a 2 expertas y a 5 profesionales en salud quienes realizaron modificaciones y sugerencias para hacerlo más comprensible al usuario; luego se llevó a cabo una prueba piloto para evaluar la validez facial en 30 usuarios de los mismos centros de atención y a partir de ésta se hicieron modificaciones para mayor comprensión del usuario.

Recolección y manejo de la información.

La información básica (nombre, edad, teléfono, seguro y fecha y hora de consulta) de todas las personas que acudieron con los diagnósticos de fiebre a estudio, virosis y dengue, fue suministrada telefónicamente por el personal de enfermería de las instituciones participantes, adicionalmente se realizó revisión de las historias clínicas del Hospital Local del Norte y Centro de salud Rosario donde se seleccionaron todas las personas que acudieron con los mismos diagnósticos. El reclutamiento se realizó mediante llamada telefónica por enfermeras hasta 3 veces, antes del tercer día luego de la fecha de consulta. En la llamada se verificaba la existencia del paciente o su acudiente y el 
diagnóstico médico, además se solicitaba consentimiento verbal y posteriormente, dos enfermeras aplicaban el instrumento de satisfacción. Para la reproducibilidad, las enfermeras de manera independiente podían aplicar el instrumento la primera o la segunda vez, pero no las dos veces, antes de 3 días contados a partir de la fecha de consulta.

La información básica y de satisfacción fue recogida en formato impreso en papel, almacenada en una base de datos con doble digitación en el programa EPI INFO 6.04 (30) y verificada mediante el subprograma VALIDATE.

Según la resolución 008430 de 1993, el presente estudio es una investigación sin riesgo. Previo a la aplicación del instrumento, se solicitó consentimiento informado verbal telefónicamente a todos los participantes.

Análisis

Se realizó un análisis de factores utilizando el modelo de máxima verosimilitud (maximum likelihood) (24), se identificaron los modelos que eran casos Heywood y se utilizó el criterio de información de Akaike (AIC) y Bayesiano (BIC) para determinar el número de factores (25), se realizó una rotación ortogonal a fin de determinar cuáles ítems tenían cargas mayores a 0,4, se utilizó la prueba de Kaiser Meyer Olkin (KMO) para evaluar la muestra (26). Se empleó el alfa de Cronbach para cada factor a fin de determinar la consistencia interna del instrumento, la direccionalidad de las preguntas y las correlaciones de cada ítem con otros ítems y con el resto del test. Para evaluar la reproducibilidad de cada ítem se utilizó el coeficiente de kappa con una ponderación al cuadrado $(23,27)$. Para evaluar la reproducibilidad general, se utilizó Coeficiente de Correlación Intraclase (CCI) dos vías (evaluador y evaluación) con el modelo de efectos aleatorios, esto es según la nomenclatura de Shrout y Fleiss, el CCI2,1 (28). También se midió el CCI2,k el cual utiliza el promedio de las calificaciones a fin de comparar y determinar la variabilidad entre los evaluadores. La evaluación del acuerdo en el método se realizó calculando los límites, como lo plantean Bland y Altman (29).

Los datos fueron analizados en el programa STATA SE ®versión 9(31).

\section{RESULTADOS}

De 205 pacientes reportados con SFA, $152(74,1 \%)$ contestaron telefónicamente, $10(4,9 \%)$ habían suministrado un número telefónico inexistente y $43(20,9 \%)$ no contestaron. De los que contestaron, 5 (3,2 \%) 
no consintieron verbalmente y $1(0,6 \%)$ no terminó la encuesta. En total se recogieron 146 encuestas entre noviembre de 2008 y agosto de 2009, el promedio de edad de los encuestados fue de 34,2 (Rango de 15 -77 años), en una razón de 4,2:1 Mujer/hombre, 49(33,6 \%) de las encuestas fueron respondidas por los propios usuarios, en los menores de 15 años, 77(52,7 \%) fueron contestadas por las madres de los usuarios y 19(13,2\% ) por otros acompañantes a la consulta como el padre, hermanos o familiares encargados. Sólo 5 personas no aceptaron responder la encuesta.

En la Tabla 1 se describen las características de la población encuestada donde se observa que $77,7 \%$ de las personas pertenecen al régimen subsidiado; $79,7 \%$ pertenecen a estratos 1 y 2, y 98,6 \% tienen algún nivel de estudio. Al comparara las características sociodemográficas por sexo se observó una diferencia estadísticamente significativa en la ocupación, con mayor frecuencia de hombres con estudios universitarios.

Tabla 1. Características socio demográficas

\begin{tabular}{|c|c|c|c|}
\hline Característica & $\begin{array}{c}\text { Mujeres } \\
n=118\end{array}$ & $\begin{array}{c}\text { Hombres } \\
n=28\end{array}$ & $\begin{array}{c}\text { Total } \\
n=146\end{array}$ \\
\hline Edad. Media(DS) & $34,1(1,2)$ & $34,9(2,6)$ & $34,2(1,1)$ \\
\hline Seguridad Social n (\%) & & & \\
\hline Subsidiado & $92(78,6)$ & $20(74,1)$ & $112(77,8)$ \\
\hline Contributivo & $1(0,8)$ & $1(3,7)$ & $2(1,39)$ \\
\hline Vinculado & $13(11,1)$ & $4(14,8)$ & $17(11,8)$ \\
\hline Sin seguridad & $11(9,4)$ & $2(7,4)$ & $13(9)$ \\
\hline \multicolumn{4}{|l|}{ Centro de Atención } \\
\hline Rosario & $14(12,1)$ & $4(14,3)$ & $18(12,5)$ \\
\hline Mutis & $30(25,9)$ & $7(25)$ & $37(25,7)$ \\
\hline Girardot & $26(22,4)$ & $7(25)$ & $33(22,9)$ \\
\hline Toledo Plata & $33(28,4)$ & $7(25)$ & $40(27,8)$ \\
\hline Hospital Local Norte & $13(11,2)$ & $3(10,7)$ & $16(11,1)$ \\
\hline \multicolumn{4}{|l|}{ Estrato (recibo de luz) } \\
\hline 1 & $41(35,6)$ & $6(21,4)$ & $47(32,9)$ \\
\hline 2 & $53(46,1)$ & $14(50)$ & $67(46,8)$ \\
\hline 3 & $18(15,6)$ & $8(28,6)$ & $26(18,2)$ \\
\hline 4 & $3(2,6)$ & 0 & $3(2,1)$ \\
\hline \multicolumn{4}{|l|}{ Nivel educativo } \\
\hline Primaria & $38(32,2)$ & $6(21,4)$ & $44(30,1)$ \\
\hline Secundaria & $59(50)$ & $15(53,6)$ & $74(50,7)$ \\
\hline Técnico & $14(11,9)$ & $3(10,7)$ & $17(11,6)$ \\
\hline Universitario & $5(4,24)$ & $4(14,3)$ & $9(6,2)$ \\
\hline Ninguno & $2(1,69)$ & 0 & $2(1,4)$ \\
\hline \multicolumn{4}{|l|}{ Ocupación * } \\
\hline Hogar & $66(55,9)$ & 0 & $66(45,5)$ \\
\hline Estudiante & $11(9,3)$ & $2(4,4)$ & $13(8,97)$ \\
\hline Empleado & $20(16,95)$ & $7(25,9)$ & $27(18,6)$ \\
\hline Independiente & $17(14,4)$ & $14(51,85)$ & $31(21,4)$ \\
\hline Desempleado & $4(3,4)$ & $4(14,8)$ & $8(5,5)$ \\
\hline
\end{tabular}


En el análisis de factores por el método de máxima verosimilitud se observó la existencia de casos Heywood con modelos que tuvieran más de tres factores, además los valores de BIC y AIC eran los menores para el modelo con 3 factores; por tanto, según este criterio se escogió el modelo de tres factores que explicaba más del $40 \%$ de la varianza y cada factor con autovalores superiores a 1 . Luego de establecer el número de factores y de realizar una rotación ortogonal, se observó el comportamiento de cada ítem, 16 ítems presentaron cargas mayores a 0,4 en un solo factor; el resto de ítems presentaban cargas con valores similares en varios factores o menores a 0,4 , por lo tanto estos ítems no entraron al modelo (Tabla 2).

Tabla 2. Análisis de Factores del instrumento de satisfacción de los serviciosde urgencias y consulta oportuna de Bucaramanga (máxima verosimilitud con rotación ortogonal)

\begin{tabular}{|c|c|c|c|c|}
\hline Ítem & Factor 1 & Factor 2 & Factor 3 & Singularidad \\
\hline 2. Examen físico del Médico & & 0,64 & & 0,45 \\
\hline 4. Amabilidad del Médico & & 0,73 & & 0,43 \\
\hline 5. Disposición del Médico & & 0,94 & & 0,10 \\
\hline 6.Respeto del Médico & & 0,65 & & 0,48 \\
\hline 7. Tiempo de dedicación a la Consulta & & 0,47 & & 0,47 \\
\hline 8. Medicamentos Formulados & & & 0,63 & 0,52 \\
\hline 9. Procedimientos de enfermería & 0,88 & & & 0,19 \\
\hline 10. Amabilidad de enfermeria & 0,80 & & & 0,33 \\
\hline 11. Agilidad de Enfermería & 0,83 & & & 0,28 \\
\hline 12. Disposición de Enfermería & 0,78 & & & 0,35 \\
\hline 13.Información por Enfermería & 0,60 & & & 0,43 \\
\hline 14. Amabilidad de la persona de facturación & & & 0,64 & 0,58 \\
\hline 22. Trámites y papeleos & & & 0,51 & 0,73 \\
\hline 23. Tiempo de espera para facturación & & & 0,71 & 0,49 \\
\hline 27. Servicio en general & & & 0,54 & 0,50 \\
\hline
\end{tabular}

Tabla 3. Valores de Kappa con ponderación al cuadrado para cada uno de los ítems

\begin{tabular}{lccc}
\hline \multicolumn{1}{c}{ Ítem } & $\begin{array}{c}\text { Acuerdo } \\
(\%)\end{array}$ & $\begin{array}{c}\text { Acuerdo } \\
\text { esperado }\end{array}$ & $\begin{array}{c}\text { Kappa } \\
\left(\mathrm{w}^{2}\right)\end{array}$ \\
\hline 2. Examen físico del Médico & 97,6 & 77,2 & 0,897 \\
\hline 4. Amabilidad del Médico & 99,3 & 77,9 & 0,970 \\
5. Disposición del Médico & 98,9 & 78,2 & 0,953 \\
6. Respeto del Médico & 88,4 & 85,9 & $0,178^{*}$ \\
7.Tiempo de dedicación a la Consulta & 96,4 & 82,4 & 0,800 \\
8. Medicamentos Formulados & 98,8 & 75,3 & 0,952 \\
9. Procedimientos de enfermería & 94,0 & 78,5 & 0,725 \\
10. Amabilidad de enfermería & 93,6 & 85,9 & $0,550^{*}$ \\
11. Agilidad de Enfermería & 94,2 & 80,8 & 0,698 \\
12. Disposición de Enfermería & 98,0 & 82,2 & 0,891 \\
13. Información por Enfermería & 98,8 & 73,2 & 0,956 \\
14. Amabilidad de la persona de facturación & 89,8 & 82,7 & $0,415^{*}$ \\
22. Trámites y papeleos & 97,3 & 88,0 & 0,781 \\
23. Tiempo de espera para facturación & 92,7 & 75,0 & 0,71 \\
27. Servicio en general & 92,0 & 65,4 & 0,768 \\
\hline${ }^{*}$ valores de Kappa Inferiores a 0,6 & & &
\end{tabular}


Adicionalmente, se observó que los factores agruparon los ítems de la siguiente manera: factor 1 atención de enfermería, factor 2 atención médica y factor 3 organización del centro médico. Para determinar si el muestreo fue adecuado, se calculó el estadístico de Kaiser-Meyer-Olkin, el cual tuvo un valor de 0,80 , considerándose un muestreo meritorio. La consistencia interna calculada mediante el alfa de Cronbach para el primer factor fue 0,89 (IC95 \% 0,86;0,93) el segundo fue 0,78 (IC95 \% 0,67;0,84) y el tercero 0,88 (IC95 \% 0,85;0,91). Se realizó reproducibilidad a 19 encuestas, el coeficiente Kappa ponderado de la sumatoria de los ítems para una reproducibilidad general fue 0,90 (IC95\% 0,77; 0,97) sin embargo, al calcular el Kappa para cada uno de los ítems, el 6, 10 y 14 presentaron una reproducibilidad deficiente, mientras que el resto de ítems presentaron un Kappa similar al general (Tabla 3).

Además la reproducibilidad también fue calculada mediante el coeficiente de correlación intraclase CCI $(2.1)$ y CCI $(2, \mathrm{k})$ con resultados de 0,95 (IC95 \% $0,82 ; 0,98)$ y 0,977 (IC95 \% 0,92; 0,99) obtenidos a partir de la sumatoria de los ítems. Para la evaluación del acuerdo se calculó el promedio de las diferencias en la sumatoria de los ítems que fue de -1,3 (DS: 8,5) y los límites de acuerdo de Bland y Altman del $95 \%$ estuvieron entre $-18,9$ y 15,3.

\section{DISCUSIÓN}

El presente estudio demuestra la validez y confiabilidad del instrumento de satisfacción para personas con SFA atendidas en los servicios de urgencias y consulta oportuna de la red pública del sistema de salud colombiano. En este estudio se observa que la satisfacción no es un constructo unidimensional debido al número de factores encontrados (satisfacción de enfermería, médica y de organización de los servicios) hallazgos similares a los mencionados en otros estudios, evidenciando la existencia entre 3 y 7 factores $(2,15,32)$. Se resalta que, 10 de los ítems del presente instrumento no lograron establecerse en algún factor; una de las hipótesis es que estos factores podrían hacer parte de otro constructo diferente al de satisfacción, ya que correspondían a la evaluación de las instalaciones y equipos médicos. Otra hipótesis es que para esta población con gran proporción en estratos socioeconómicos bajos y pertenecientes en su mayoría al régimen subsidiado, las instalaciones no impactan de igual manera en la satisfacción como lo hace la atención de enfermería, médica o la organización del centro de atención; concordando con otros hallazgos donde las dimensiones no pesan igual al evaluar la satisfacción global (15). 
Por otro lado, la consistencia interna de cada uno de los factores encontrados es considerablemente alta $(0,78-0,89)$ lo que demuestra que las preguntas planteadas para cada factor realmente corresponden a dicho factor y confirman la multidimensionalidad del constructo.

También este instrumento presenta una buena reproducibilidad $(>0,90)$ ya sea calculada por el método del Kappa de Cohen ponderada o por Coeficiente de Correlación intraclase, sin embargo, al evaluar la reproducibilidad de cada uno de los ítems, se observa que tres de estos presentan valores muy bajos (respeto del médico, amabilidad de la enfermera y amabilidad de la persona de facturación) una posible explicación es que haya faltado poder para evaluar su reproducibilidad. Al comparar los coeficientes de correlación intraclase 2.1 y 2 .k se observa una gran similitud, lo que indica una baja variabilidad entre las encuestadoras.

Existen varias metodologías para medir la satisfacción del paciente como los buzones de sugerencias, las quejas formales, las encuestas, entre otros; estas últimas son el método más utilizado, siendo aquellas administradas telefónicamente y las suministrados persona a persona las que proporcionan mayor tasa de respuesta con respecto a otras opciones como los cuestionarios autodiligenciados o enviados por correo (9); pese a esto, una de las limitaciones del sistema telefónico que se observó en este estudio, es que las personas de estratos socioeconómicos bajos no siempre cuentan con servicio telefónico fijo o móvil por lo que podría darse un sesgo de selección; sin embargo, el contacto telefónico pueden hacer sentir en los usuarios que las prestadoras de salud tienen un mayor grado de interés en sus opiniones que cuando ésta se realiza de manera auto diligenciada, por lo que su vez puede mejorar el nivel de satisfacción.

En Colombia según el decreto 1011 de 2006, las Empresas Administradoras de Planes de Beneficios (EAP) y los Prestadores de Servicios de Salud mediante la auditoría, deben evaluar obligatoria y sistemáticamente la satisfacción de los usuarios con respecto al ejercicio de sus derechos, al acceso, la oportunidad y a la calidad de sus servicios (33). El Ministerio de la Protección Social establece mediante la resolución 1446 de 2006, 28 indicadores de calidad para el SOGCS, de los cuales 3 evalúan la satisfacción del usuario, estos son: proporción de quejas resueltas antes de 15 días, tasa de traslados y satisfacción global; para este último, establece como mecanismo de medición las encuestas de satisfacción de los usuarios; pero, la resolución no determina el tipo de encuesta a utilizar, por lo que cada entidad es libre de 
aplicar el instrumento de su interés sin conocer si este es válido o no (10). Por otro lado, la encuesta nacional de salud (ENS) 2007 reportó una satisfacción del paciente por encima del $70 \%$ en las entidades públicas y del $80 \%$ en las privadas; pese a esto, no se describe el uso de un instrumento válido para tal fin (21).

Lo anterior, hace suponer la utilización indiscriminada y variada de instrumentos para medir satisfacción sin que hayan sido sometidos a pruebas de validez y confiabilidad, y por lo tanto los resultados obtenidos podrían no reflejar la realidad de la satisfacción del usuario y dificultar la comparabilidad; lo cual se puede ver traducido en la toma de decisiones equivocadas generadas a partir de la evaluación de los servicios; por lo tanto, es importante que se realice unificación de criterios y se utilicen instrumentos válidos y reproducibles a fin de lograr una estandarización de la evaluación de la satisfacción y la comparabilidad de los resultados obtenidos entre las diferentes instituciones o servicios.

En conclusión, existe la necesidad de aplicación de instrumentos válidos y reproducibles que evalúen satisfacción del usuario en los diferentes servicios y que permitan la comparabilidad, teniendo en cuenta las características propias del sistema de salud y de la población (34)

Agradecimientos: A Luis Carlos Orozco Vargas. Médico, Mg Epidemiología, Universidad Industrial de Santander. A Juan José Rey, Médico, Mg Epidemiología. Gerente de la ESE ISABU. Al personal de enfermería de la ESE ISABU.

\section{REFERENCIAS}

1. Donabedian A. An introduction to quality assurance in health care. Oxford University Press; 2003. $p 47$.

2. Andaleeb S. Service quality perceptions and patient satisfaction: a study of hospitals in developing countries. Soc Sci Med 2001; 52: 1359-1370.

3. Donabedian A. Special article: The quality of care: how can it be assessed? archives of pathology \& laboratory medicine. Nov. 1997; 121(11): 1145.

4. Zastowny TR, Roghman KJ, Cafferata GL. Patient satisfaction and the use of health services. MedCare. 1989; 27:705-23.

5. Ramirez T, Nájera A, Nienda G. Percepción de la Calidad de los servicios de salud en México: perspectiva de los usuarios. Salud Publica Mex.1998; 40:3-12.

6. Jiménez R. Indicadores de calidad y eficiencia de los servicios hospitalarios. Una mirada actual. Rev Cubana Salud Pública. Ene-mar. 2004; 30 (1).17-36.

7. Fitzpatrick R. Surveys of patient satisfaction: I-important general considerations. BMJ 1991; 302: 887-889.

8. Boudreaux ED, D'Autremont S, Wood K, Jones GN. Predictors of emergency department patient satisfaction: stability over 17 months. Acad Emerg Med. 2004 Jan;11(1):51-8. 
9. Sitzia J, Wood N. Response rate in patient satisfaction research: an analysis of 201 published studies. Int J Qual Health Care. 1998; 10: 311-317.

10. Sistema de Información para la Calidad. Resolución 1446 del 8 de mayo. Ministerio de la protección social. 2006. Disponible en: http://mps.minproteccionsocial.gov.co/ vbecontent/NewsDetail.asp?ID=15504\&IDCompany=11 Consultado: enero 2011.

11. Trowt A, Magnuson R, Hedges J. Patient satisfaction investigation and emergency department: What does Literature say?. Academic Emergency Medicine. Jun 2007;7(6):695709.

12. Van Campen C, Sixma H, Friele RD, Kerssens JJ, Peters L. Quality of care and patient satisfaction: a review of measuring instruments. Med Care Res Rev. 1995; 52: 109133.

13. Quintana JM, Aróstegui I, Arcelay A, García M, Ortega M, Candelas S. Patient satisfaction survey on emergency departments in acute care hospitals. Gac Sanit. 1999 JanFeb;13(1):38-45.

14. Hernan M, Jiménez JM, March JC, Silió F. Calidad percibida por los clientes del Hospital Costa del Sol. Granada: Escuela Andaluza de Salud Pública, 1996.

15. Serrano R, Loriente N [Internet]. The anatomy of patient satisfaction. Salud pública Méx. 2008; 50(2): 162-172.

16. García X, Prieto CA, Milachay M, Rangel MC. Evaluación del grado de satisfacción de los pacientes atendidos en el postgrado de ortodoncia de la Universidad del Bosque durante los años 1999-2003. Revista Científica. 2004;10(1):50-63.

17. Roldán $P$, Vargas CR, Giraldo CP, Valencia GE, García CL, Salamanca L M. Evaluación de la calidad en la atención en salud. Un estudio de caso. Colombia Médica. 2001;32(1):14-18

18. Bonilla JA, Sanín A, Pedraza P, García A, Sanchez C. Evaluación de la técnica anéstésica con remifentanilo y midazolam en el legrado Obstétrico. Rev Col Anest, jul-sep 2007; 35(3):215-219.

19. Eslava-Schmalbach J, Gaitán-Duarte H, Gómez-Restrepo C. A scale for measuring the quality of post-anaesthetic recovery from the usuries point of view. Rev. salud pública (Bogotá) 2006; 8(1):52-62.

20. Rade BV. Customer satisfaction. International Journal of Health Care Quality Assurance. Bradford: 2006.19(1):8-305.

21. Encuesta Nacional de Salud ENS 2007. Colombia. COLCIENCIAS-Ministerio de la Protección Social. Enero de 2009.

22. Observatorio de Salud pública. Indicadores de Morbilidad basados en el registro individual de servicios de salud (RIPS). Revista del Observatorio de Salud Pública 2007; 3(2): Sup 1.

23. Orozco L. Medición en Salud. Medición en salud: Diagnóstico y evaluación de resultados, un manual más llá e lo básico. 1 edición. Bucaramanga: División de publicaciones Universidad Industrial de Santander; 2010.

24. Tucker L, Lewis Ch. A reliability coefficient for maximum likelihood factor analysis. Psychometrika 1973;38( 1):1-10.

25. Jones A, O'Donnell O. Econometric analysis of health data. Chichester: John Wiley \& Sons Ltd.; 2002. p. 90.

26. Norman JG, Strainer DL. Bioestadística. Madrid, Mosby/ Doyma;1996.

27. Fleiss JL, Cohen J. The equivalence of weighted kappa and the intraclass correlation coefficient as measures of reliability. Educational and Psychological Measurement. 1973; 33:613-619.

28. Shrout PE, Fleiss JL. Intraclass correlations: Uses in assessing rater reliability. Psychological Bulletin1979; 86:420-428.

29. Bland JM, Altman DG. Comparing two methods of clinical measurement: A personal history. International Journal of Epidemiology. 1995; 24(suppl. 1):S7-S14. 
30. CDC. Epi Info. versión 6.04d. Epidemiología en ordenadores.Atlanta, Georgia; Enero, 2001.

31. Stata Corp. Stata Statistical Software: Release 9.2. College Station, Texas USA; 2006.

32. Nathorst-Boos J, Munck I, Eckerlund I, Ekfeldt Sandberg C. An evaluation of the QSP and the QPP: two methods for measuring patient satisfaction. International Journal for Quality in Health Care 2001; 13(3)pp 257-264.

33. República de Colombia. Decreto 1011 de 2006 Sobre el Sistema Obligatorio de la garantía de la Calidad del Sistema General de Seguridad Social en Salud SOGCS.

34. Crow R, Gage H, Hampson S, Hart J, Kimber A, Storey L, et al. The measurement of satisfaction with healthcare: implications for practice from a systematic review of the literature. Health Technol Assess 2002; 6(32): 1-245. 


\section{Apéndice 1}

\begin{tabular}{|c|c|}
\hline $\begin{array}{c}\text { ENCUESTA TELEFÓNICA DE SATISFACCIÓN } \\
\text { UIS-ISABÚ } \\
\text { SERVICIO DE URGENCIAS- CONS. OPORTUNA }\end{array}$ & \multicolumn{1}{c|}{ COD } \\
\hline \multicolumn{2}{|c|}{ ESCALA DE SATISFACCIÓN DEL USUARIO } \\
\hline
\end{tabular}

A continuación le haremos una serie de preguntas para saber cuan satisfecho está usted con la atención recibida en urgencias. Recuerde que todas sus respuestas son confidenciales y su nombre no será revelado.

De la manera más honesta posible, califique de 0 a 10 que tan satisfecho está usted con la atención que le dieron la ultima vez que fue a urgencias. Marque con una $\mathbf{X}$ la casilla correspondiente según su grado de satisfacción. 0 equivale a nada satisfecho y 10 sería totalmente satisfecho, la minima calificación es 0 y la máxima es 10

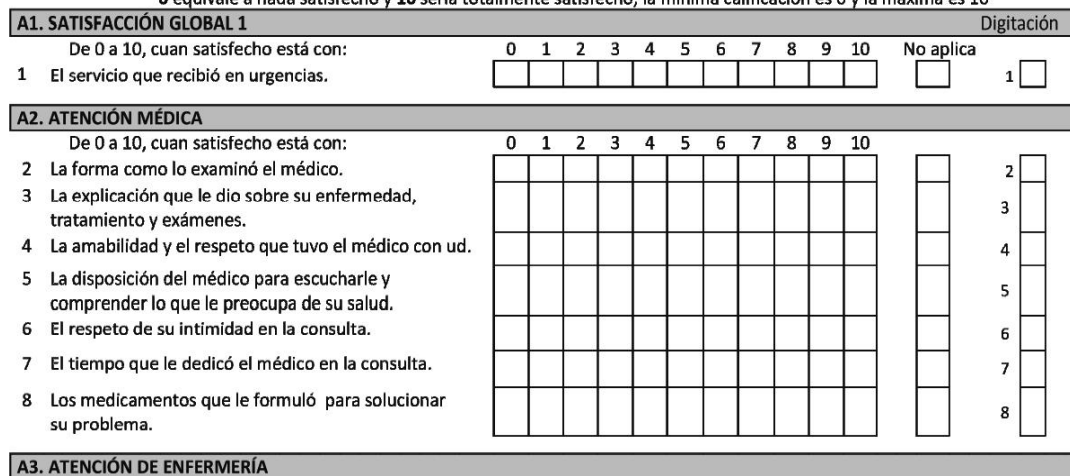

A3. ATENCIÓN DE ENFERMERÍA

De 0 a 10 , que tan satisfecho está con:

9 Los procedimientos que realizó enfermería.

10 La amabilidad y el respeto que tuvo enfermería con ud.

11 La agilidad y práctica del personal de enfermería.

12 La disposición de enfermería para escucharle y

comprender lo que le preocupa de su salud.

13 La información que le dio sobre los cuidados que debe tener en casa.

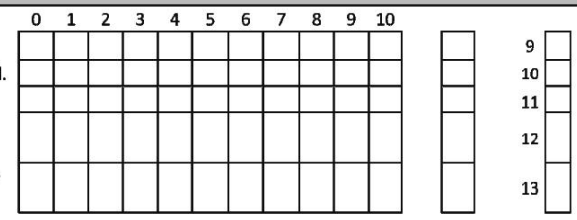

A4. PERSONAL ADMINISTRATIVO

De 0 a 10 cual es su satisfacciónc

La amabilidad y el respeto que tuvo la persona de facturación (ventanilla).

15 La amabilidad y el respeto que tuvieron los celadores.

16 El trato que dieron a sus familiares.

\section{A5. INSTALACIONES}

De 0 a 10 , cuan satisfecho está con:
La limpieza y el orden del centro de salud u hospital.

18

18 La comodidad de la sala de esp

19 La comodidad del consultorio.

20 El estado de las paredes, techos, puertas y ventanas.

21 Los equipos médicos disponibles para su atención.

\section{A6. ORGANIZACIÓN}

De 0 a 10, cuan satisfecho está con: $\quad 0 \begin{array}{llllllllllll}0 & 0 & 1 & 2 & 3 & 4 & 5 & 6 & 7 & 8 & 9 & 10\end{array}$

22 Los trámites y papeles que tuvo que hacer para

que lo atendieran.

23 El tiempo que tuvo que esperar para que le

facturaran la consulta.

$24 \quad$ El tiempo que tuvo que esperar para

que lo atendiera el médico.

25 La forma como el centro suministra los

medicamentos.

26 La forma como el centro realiza los exámenes.

A7. SATISFACCIÓN GLOBAL 2

De 0 a 10, cuan satisfecho está con:

27 El servicio que recibió en urgencias.

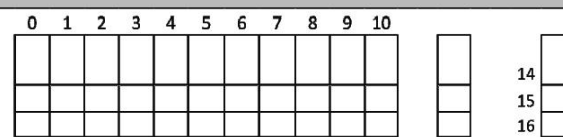

28 ¿Recomendaría este servicio a sus familiares y amigos?

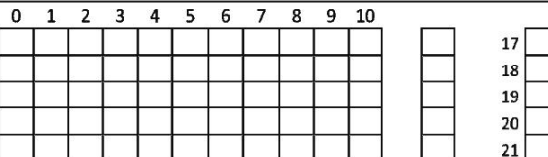

\begin{tabular}{l|l}
17 & $\square$ \\
18 & \\
19 & \\
20 & \\
21 &
\end{tabular}

Le agradecemos por su colaboración y tiempo al responder esta encuest

Nombre del encuestador 